\title{
Neue Medien - Erste Anmerkungen zu einer Herausforderung
}

\author{
von Gerburg E. Vogt
}

Ich bin in eine Gegend und einige Bergbauernhöfe verliebt. Dort verbringe ich jeden Sommer ein paar Wochen einfachen Lebens mit wenig Komfort und viel Kontakt mit einfachen Leuten. Ein solcher Wechsel von der Großstadt in die beinahe reine Natur mit Wasser vom Brunnen und Holzfeuer setzt jedesmal erneut Denk- und Uberprüfungsprozesse in Gang, die heilsam relativierend, ernüchternd in jeder Hinsicht sind. Dies bemerke ich beiläufig auch bei Bekannten und Freunden, die sich anschließen. $\mathrm{Da}$ bleiben manchmal auch Schocks nicht erspart wie z. B. der folgende: Auf dem Heimweg von einer Bergtour überrascht uns ein Gewitter. Wir laufen die letzten 500 Meter quer über die Wiesen auf ein kleines Haus zu, in dem ebenerdig in zwei Stuben die Witwe Maria wohnt. Wir kennen uns seit Jahren. Sie hat für vieles einen Rat und kann aus nichts etwas machen. Wir kommen patschnaß bei ihr an. Sie macht die Ofenbank frei, daß wir uns wärmen können, holt eine Flasche und schenkt jedem von uns zwei Schluck brauner Flüssigkeit ein. Das sei ausgezeichnet und bewahre uns vor einer Erkältung. Einer aus der Gruppe, ein Erstling hier heroben, fragt nach der Zusammensetzung und dem Rezept. Maria erzählt gern, wie sie im Sommer anfängt, diesen Schnaps vorzubereiten. „Und das wissen Sie sicher noch von früher, von Ihrer Großmutter oder so, wie man so etwas macht", fragt unser Neuling. "Na, na", meint Maria, „diese Rezepte gibt jede Woche die Frau Dr. Sowieso in der Stunde der Hausfrau im Radio Bozen."

Für unseren Nostalgiker war das ein harter Schlag. Er hätte so gern gehabt, daß wenigstens dort oben die Welt noch in Ordnung gewesen wäre, ohne Massenmedien, ohne Technik, ohne Beeinflussung, ohne Fremdbestimmung.

Wenn wir noch einen Moment bei diesem einfachen, unmittelbar erlebten Beispiel bleiben. Das Transistorradio in der Stube der alten Frau im Gebirge verbindet diese mit der übrigen Welt. In Marshall McLuhans Terminologie findet hier eine Ausweitung des zentralen Nervensystems zum Informationsnervensystem statt. ${ }^{1}$ Wie wäre es nun, wenn nicht nur eine Atmosphäre unmittelbarer Kommunikation, wie dies durch Hörfunksendungen geschieht, entstünde, sondern tatsächlich Kommunikation zustande käme? Wie wäre es, wenn Maria ein Telefon hätte? Ja, das wäre schön. Sie könnte die Kinder in der Stadt erreichen und den Arzt. Vielleicht würde der Enkelsohn Andreas noch leben, wenn er rechtzeitig eine Tetanusspritze bekommen hätte.

All diese Vorteile nutzen wir wie selbstverständlich. Einige möchten auch zurück oder zumindest stehen bleiben. Denn die Angst vor dem, was da neu geschaffen wird, wächst. Die sozialen Folgen sind nicht mehr zu überblicken. Die Schreckensbilder vom isolierten, kontrollierten, manipulierten Menschen vermehren sich.

Dr. phil. Gerburg Vogt SAC ist Leiterin des Projekts Bildschirmtext der Zentralstelle Medien der Deutschen Bischofskonferenz und Lehrbeauftragte für Kommunikationswissenschaft an der Hochschule für Philosophie in München. 


\section{Wovor wir Angst baben}

Zeitungsartikel, Broschüren, Bücher, Tagungsthemen signalisierten im Verlauf des letzten Jahres eine verstärkte Diskussion der zunehmenden Computerisierung unserer Lebenswelt. TIME-Magazin brachte den Computer im Januar-Heft 1983 als Maschine des Jahres. ${ }^{2}$ Und der Club of Rome, der es sich zur Aufgabe gemacht hat, über Gefährdungen nachzudenken, denen die Menschheit heute ausgesetzt ist, hat eine Dokumentation herausgegeben, die einer breiten Offentlichkeit nahebringen soll, was die Entwicklung der Mikroelektronik für die Menschheit bedeutet. „Damit soll eine durch Sachkenntnis geprägte Diskussion darüber angeregt werden, welche Wege einzuschlagen sind, damit diese neue Technik der Menschheit Vorteile und nicht etwa Nachteile bringt. "s Während sich der Durchschnittsmensch noch mit Einzelthemen wie Kabelfernsehen, Videotext und Bildschirmtext herumschlägt, tut sich bereits ein viel weiteres Spektrum der Entwicklung auf, von dem selbst Fachleute nicht wissen, ob es sich um eine neue industrielle Revolution oder „ob es sich bloß um eine weitere technische Entwicklung handelt, die gewiß bedeutsam ist, die aber reibungslos von unserer Gesellschaftsstruktur absorbiert werden kann"

Warum ist diese Frage jetzt so dringlich geworden? Haben wir uns nicht schon längst an die buchführenden und büroorganisierenden Maschinen gewöhnt, ohne die wir nicht mehr zurechtkämen? „Wenn allein die Telefonverbindungen noch per Hand geschaltet würden, müßte die ganze Bevölkerung einschließlich der Kinder stöpseln. " ${ }^{\text {S }}$ Ohne jede Übertreibung kann festgestellt werden, daß ohne Informationssysteme, wie sie die Computer darstellen, weder unsere Produktion noch unsere Verwaltung aufrechtzuerhalten wären. Die Probleme im Bereich der Information und der Kommunikation verdoppeln sich dadurch, daß Mikroelektronik und Informationstechnik sich weitgehend überschneiden und sowohl für die Massenmedien als auch die Medien des individuellen Gebrauchs, wie das Telefon und die Datenübertragungsnetze, revolutionierend zu sein scheinen.

\subsection{Isolation}

„Es wird immer deutlicher und unmißverständlicher, ... daß wir an die Grenzen dessen stoßen bzw. bereits gestoßen sind, was der Mensch um seiner selbst willen mit sich machen und geschehen lassen darf. Wo der Verlust sozialer und kommunikativer Beziehungen, wo Entmenschlichung droht, da ist das Maß voll. Spätestens dann, also jetzt, gilt es $\mathrm{zu}$ erkennen, daß der eindimensionale Fortschrittsglaube, dieses Dogma der Automatik, Autonomie und Neutralität des wissenschaftlich-technischen Fortschritts, das die gesellschaftliche Entwicklung seit mindestens eineinhalb Jahrhunderten prägt und dem neben ihren bürgerlichen Gegnern auch die traditionelle Linke noch immer anhängt, überwunden werden muß."

Aus diesem Zitat geht hervor, daß die Furcht vor dem Verlust direkter Kontakte von Mensch zu Mensch immer mehr zunimmt, daß das Gespräch am Bankschalter wegfällt, weil man sich der bequemen Einrichtung home-banking bedienen kann, daß der Plausch im Laden um die Edke nicht mehr stattfindet, weil ich meinen Salatkopf und die fünf Eier, die ich brauche, über Bildschirmtext bestellen kann. Das wäre in der Tat schade. Ich habe mir von einem „Fräulein vom Amt“ erzählen lassen, wie viele Menschen sich telefonisch wecken lassen, nur um am Morgen eine menschliche Stimme zu hören und dafür pro Weckauftrag 2,- DM aufzuwenden bereit sind. Isolation als Folge technischer Veränderungen? 


\subsection{Kontrolle total}

Noch erschreckender sind die Visionen George Orwells, der die Isolation ergänzt sieht durch die totale Kontrolle der Menschheit. Befürchtungen über die Verletzung der Privatsphäre und mißbräuchliche Verwendung von gesammeltem Material gibt es schon, seit Computer in den Verwaltungen eingesetzt werden. „Bis vor kurzem konnte man in vielen Ländern mit einiger Sicherheit davon ausgehen, daß Individuen den verschiedenen Verwaltungsstellen nur in einer relevanten Eigenschaft bekannt waren, also z. B. entweder als Steuerzahler oder als Patient oder als Schüler oder als Angestellter. In automatisierten Informationssystemen könnten Daten aus verschiedenen Quellen sehr leicht zusammengezogen werden, wenn keine Vorkehrungen getroffen werden. Wie ein Puzzlespiel würden sie ein ziemlich vollständiges, aber dennoch verzerrtes Persönlichkeitsbild ergeben. Mit der besseren Beherrschung der EDV und insbesondere mit EDV-Verbundsystemen wächst diese Gefahr. ${ }^{\alpha 7}$ Private Daten sind: Name, Geburtstag, Anschrift, Familienstand, Einkommen, Steuerklasse, Automarke, Konfessionszugehörigkeit, Krankheit, Rentenanspruch, Warenbestellung, Berufsausbildung, ehrenamtliche Tätigkeit, Urlaubsziel.

Warner Thorns gibt in seinem Buch über die Computer Society einen fiktiven Uberblick über den Tagesablauf und die Lebensgewohnheiten eines 38jährigen Programmierers, der aufgrund seiner bargeldlosen Zahlungsweise an vielen Stellen Spuren hinterläßt, die sich zu einem Gesamtbild zusammensetzen lassen. „Besitzt Aktien (90\% Wahrscheinlichkeit); schweres, kräftiges Frühstück; vermutlich Mann mit Übergewicht; für 3 Dollar Benzin gekauft; besitzt VW; bisher in dieser Woche für 12 Dollar Benzin gekauft; offenbar weitere Fahrten außer den 9 Meilen zum Arbeitsplatz; kaufte Benzin um 7 Uhr 57; anzunehmen, daß zu spät zur Arbeit gekommen.

Telefonate: Nr. 328-1826 gehört Shady Lane - Shady war 1972 wegen Wettvergehens verhaftet; Nr. 308-7928 teurer Herrenfriseur - spezialisiert auf Kahlköpfige und Modehaarschnitt; Nr. 421-1931 Bestellungen für Las Vegas (ohne Ehefrau). Werden Akten prüfen, ob jemand zu denselben Zeiten nach Las Vegas fuhr, und dies mit seinen Anrufen vergleichen. ${ }^{* 8}$

Dieses zwar konstruierte Beispiel zeigt jedoch, daß jedes gesellschaftliche Handeln einer Person, ob dies Einkauf oder Verkauf, Kinobesuch oder Tanken heißt, Informationen vermittelt, die interpretiert werden können, wie man sieht, nicht nur zum Nutzen des Betroffenen.

Der Zusammenhang zwischen neuen Medien und datenverarbeitenden, datenspeichernden und datenkombinierenden Systemen braucht wohl nicht mehr deutlicher gemacht zu werden. Ein einzelner Artikel reicht nicht aus, um diese zusätzliche Problematik hinreichend zu bearbeiten. Dieses Thema muß weiterverfolgt werden. Die Vorschläge, die in dieser Richtung von namhaften Autoren gemacht werden, sind besonders daran zu messen, wie wirksam sie den Isolierungs-, Verplanungs- und Uberwachungsängsten entgegenzuwirken imstande sind. Die Autoren des Berichts an den Club of Rome beschließen allesamt ihre Expertise mit dem Hinweis auf den noch offenen Ausgang dieses Prozesses einerseits und auf die Notwendigkeit, zu neuen Ansätzen des Denkens zu kommen, da aus diesem „komplizierten Geflecht aus VerheiBung und Zweifel ... konventionelle Weisheiten, die Politik des status quo und lineares Denken nicht den Durchbruch zu der neuen Gesellschaft bringen" kann, die prinzipiell möglich ist. ${ }^{9}$

Auch die Stellungnahme der Zentralstelle Medien der Deutschen Bischofskonferenz hat gerade auf diese offenen Fragen hingewiesen. Sie sieht sowohl die Frage nach der 
Abnahme der Primärerfahrungen und der Verkümmerung der direkten Kommunikation unter den Menschen, als auch die technisch und strukturell bedingte Eigendynamik solcher datenspeichernder und verarbeitender Systeme weder durch ausländische Erfahrungen noch durch verläßliche Aussagen im eigenen Land so beantwortet, daß verantwortliche Entscheidungen für die Zukunft getroffen werden können. ${ }^{10}$ Gerade diese Unsicherheit, was die zukünftige Entwicklung anbelangt, die zum Teil durch Vorhandenes bereits in eine gewisse Richtung gelenkt worden ist, weil die Datenkommunikation für Industrie, Handel, Verwaltung und Wissenschaft bereits lebensnotwendig ist, erzeugt Angste, die zu Frontenbildung und Meinungskampf führen. ${ }^{11}$

Wie deutlich sind die Konsequenzen absehbar, wieviel an Ungewißheit hängt vor allem damit zusammen, daß wir auf das völlig Neue niemals wirklich vorbereitet sein können. Eric Hoffer sieht „daß eine Bevölkerung, die einer drastischen Veränderung unterworfen ist, zu einer Bevölkerung von Nichtangepaßten wird, und Nichtangepaßte leben und atmen in einer Atmosphäre leidenschaftlichen Gespanntseins. Zwischen Mangel an Selbstvertrauen und gefühlsbetonter Einstellung besteht ein enger Zusammenhang; leidenschaftliche Intensität kann, wie sich zeigen wird, sehr wohl eine Art Ersatzempfindung für Selbstvertrauen sein. Dieser Zusammenhang läßt sich in allen Lebensbezirken beobachten. ${ }^{\text {12 }}$

\section{Die Angst vor dem Neuen}

Ein guter Bekannter, ein Professor, versucht seinen Studenten immer wieder zu vermitteln, daß Erfahrung dumm mache. Diese Behauptung begründet er mit dem Hinweis, daß Gewohnheiten, eingefahrene Verhaltensweisen, Routine es dem Menschen schwer machen, Veränderungen herbeizuführen, neue Wege oder Verfahren zu entdecken und anzuwenden. Eine ähnliche Behauptung stellt Karl W. Deutsch auf, wenn er dem Ubergang vom "kindlichen" zum "erwachsenen“ Lernen einen Zug ins Pathologische zuschreibt. ${ }^{13} \mathrm{Er}$ meint damit das Eingefahrensein auf bestimmte Lösungen, die sich bewährt haben. Bewährt haben sich, um im Bereich der Information zu bleiben, die Zeitungen, die Zeitschriften, die Bücher. Bewährt hat sich das Telefon. Bewährt hat sich der allgemeine Rundfunk. Das können wir heute sagen. Wie aber haben sich diese Techniken, diese Medien, dieses Bewährungszeugnis verdient? Zunächst einmal haben wir uns an sie gewöhnt. Sie gehören zu unserem alltäglichen Leben. Sie sind uns nicht mehr neu.

\subsection{Zwei oder drei geschichtliche Beispiele}

In der Zeit ihrer Entstehung waren sie weder unumstritten noch selbstverständlich. Sie wurden nicht nur als Fortschritt und Segen für die Menschheit betrachtet. Jede Kulturtechnik erschien vielen zunächst als ein Eingriff in die Natur des Menschen, als ein Mittel, den Menschen zu entmenschlichen, ihm seine ureigensten Fähigkeiten zu rauben und ihn ärmer zu machen. Hat das die Erfindung des Alphabets getan? Socrates z. B. meint, daß sie in der Seele der Lernenden Vergeßlichkeit bewirke, "weil sie nicht mehr ihr Gedächtnis gebrauchen werden, sondern auf geschriebene Buchstaben außerhalb ihrer selbst vertrauen werden und sich nicht mehr an sich selbst erinnern werden ... Du gibst deinen Schülern nicht die Wahrheit, sondern nur ein Abbild der Wahrheit. Sie werden Helden vieler Dinge sein und werden doch nichts gelernt haben. Sie werden allwissend erscheinen und doch aufs Ganze gesehen überhaupt nichts wissen "14. Was Socrates an Befürchtungen der Schrift gegenüber hatte, entspricht unseren Be- 
fürchtungen den technischen Hilfsmitteln der heutigen Schüler gegenüber. Allerdings addiert sich der Taschenrechner zu anderen vorhandenen Medien und verändert die Art und Weise des Lernens. Und nicht nur das.

Die massenhafte Verbreitung gedruckter Flugblätter, Pamphlete und Zeitungen brachte nicht nur Nachrichten und Neuigkeiten, Handelsinformationen oder religiöse Angriffs- und Verteidigungsschriften. Sie bewirkte eine Veränderung der gesellschaftlichen Situation. Die neue Möglichkeit, Gedanken, Ideen, Meinungen auszutauschen und zu verbreiten, war nicht mehr auf kleine Gruppen, auf wenige Handelspartner, auf Fürstenhäuser oder gebildete Bürger begrenzt. Die Teilnehmer wurden immer zahlreicher und die Besorgnisse der Herrschenden immer größer: „Wenn der Teufel von Gott die Ermächtigung erhalten könnte, eine sichtbare Form zu wählen zur besseren Förderung seiner Tätigkeit auf dieser Welt, dann würde er sich als Zeitung produzieren. ${ }^{\text {"15 }}$

Will man die Erfindung der Schrift als erste Revolution des Kommunikationsprozesses bezeichnen und den Buchdruck als zweite, dann ist das Telefon als distanzüberwindendes Kommunikationsinstrument zwischen zwei Menschen der Anfang der dritten Revolution. Die New Yorker "Daily Graphic" vom 15. März 1877 brachte auf ihrem Titelblatt „Die Schrecken des Telefons - Die Redner der Zukunft.“ Ein Svengali steht mit fliegenden Haaren vor einem Mikrofon in einem Studio und peitscht die Massen auf. Dasselbe Mikrofon wird in London, San Francisco, in der Prärie und in Dublin gezeigt. Seltsamerweise sah die Zeitung von damals im Telefon eine Konkurrenz der Presse als Public-Address-System, wie es das Radio fünfzig Jahre später tatsächlich sein sollte. ${ }^{16}$ Das erste Telefon-Teilnehmer-Verzeichnis in Berlin wurde dort das Verzeichnis der hundert Irren genannt. Die Unsicherheit der neuen Möglichkeit gegenüber hat sich in zahlreichen Witzen und humorvollen Dialogszenen Luft gemacht.

Die nachfolgenden Entwicklungen der Elektroindustrie wurden zwar noch immer mit Staunen, aber schon mit einer gewissen Selbstverständlichkeit angenommen. Das Grammophon meines Großvaters war noch eine Attraktion für die Nachbarn und ein Stein des Anstoßes für den Pfarrer, der sich nach der ersten Einladung zu einem Konzert jedoch von der Vereinbarkeit mit einem christlichen Lebenswandel überzeugen ließ. Der Radiaoapparat meiner Eltern gehörte zur Spitzenklasse, der auf internationale Frequenzen einzustellen war. Unter den Nazis wurde er meinem Vater zum Verhängnis.

Die Zeit, in der architektonische Neuheiten den Namen Citta 2000 erhielten und auch technische Geräte mit dem Etikett der Vollendung des Jahrhunderts in die Zukunft wiesen, scheint vorbei zu sein. Unsicherheit, Angst, Pessimismus, Resignation haben viele Menschen den offenen oder heimlichen Rückzug antreten lassen, um zu retten, was noch zu retten ist.

Andererseits befindet sich der Mensch „auf dem Weg in die Grenzenlosigkeit. Welch ein Wandel binnen weniger Jahrzehnte, welche Dynamik der letzten drei bis vier Generationen angesichts einer vielleicht sechzig- bis hundertausend Jahre weit zurückreichenden, von Menschen bewußt erlebten Geschichte. Ein einziges Jahr unserer Zeit gebiert mehr Wissen für die Zukunft der Menschen als ein ganzes Jahrtausend der Frühgeschichte ... Die Zeitenwende hat längst stattgefunden. Sie weist den Menschen eine andere, ungewohnt eigentümliche Rolle zu: Es heißt Abschied nehmen von der Vorstellung, Menschen könnten wie seit alten Zeiten in körperlicher Präsenz Entdecker oder gar Eroberer ferner Gebiete sein. Zu groß sind die Räume, zu weit ent- 
fernt sind fremde Sterne. Wir sind Kinder dieser Erde ... Technische Automaten, Roboter, Raumsonden mïssen unsere Stellvertreter und Botschafter auf dem Weg in die Grenzenlosigkeit sein. Uns bleibt, daheimgeblieben, die geistige Auseinandersetzung mit Forschungserkenntnissen, nicht die körperliche Besitznahme. "17 So kennzeichnet Claus Henninger aus der Perspektive der Forschungsarbeit für die Raumfahrt die Rolle des Menschen an der Wende zum 21. Jahrhundert und seine Aufgabe im Umgang mit dem Wissen, von dem selbst die Beteiligten höchstens Ahnungen haben können.

\section{Was tun?}

Es ist bekannt, daß konkretes Wissen über eine Sache die Einstellung zu ihr stärker stabilisiert, als wenn nur Vermutungen über sie existieren. Was aber wissen wir konkret z. B. über die Auswirkung Neuer Medien und den Umgang der Menschen damit. Claus Eurich stellt zu Recht fest, „daß das eine oder andere Problem bzw. das gesamte Problemspektrum sich unter heute nicht absehbaren Ereignissen und Bedingungen morgen ganz anders darstellen kann"18. Er hat auch recht, wenn er fordert, daß diese Prognosenunsicherheit nicht von den notwendigen Konsequenzen ablenken und abhalten sollte.

Nur, wo ist zuerst anzusetzen? Etwa beim sozialen Wohnungsbau, daß die Großväter und Großmütter wieder Platz finden und nicht mehr zum Trost Fernsehen müssen, sondern mit ihren Enkelkindern spielen können? Bei der Verhinderung von Bildschirmtext, der an sich eine Reihe von Anwendungsmöglichkeiten und brauchbare Informationen leicht zugänglich anbieten kann, die man sonst mühsam zusammensuchen muß? Wohin kann ich mich z. B. in einer Großstadt wenden, wenn ich eine Schulaufgabenüberwachung, eine zeitweise Unterbringung für einen behinderten Menschen, einen Mahlzeitendienst für eine alte Nachbarin suche? Die Liste der Informationsdefizite könnte noch verlängert werden. Wer hat nicht schon Behördengänge umsonst gemacht, weil er ein Dokument zu wenig dabei hatte. Wer hat nicht schon unfreundlichen aber direkten Kontakt mit Beamten gehabt, der sich durch mehr Vorwissen, Vorinformation erübrigt hätte?

In der Auseinandersetzung um Chancen und Risiken neuer Medien wird vor allem der Rückgang an direkter Kommunikation befürchtet. Claus Eurich beklagt die Vergeßlichkeit der Menschen, sobald eine neue Technologie auftauche. Sofort wende man sich ihr zu und berücksichtige nicht mehr, was Generationen von Sozialwissenschaftlern, Kleingruppen über Nachbarschaften bis zu Dorfgemeinschaften zusammengetragen hätten. ${ }^{19}$

\subsection{Gute, alte Zeit: nur gut?}

Es bleibt natürlich auch zu fragen, ob denn alles so ideal ohne Fernsehen war. Wer hat an den Gesprächen am Dorfbrunnen teilgenommen? Welche Themen wurden behandelt? Wieviel Zerstörung menschlichen Glïcks, Ächtung Andersdenkender, Isolierung Armer, sozial Schwacher, wieviel sozialen Druck haben solche Kommunikationsvorgänge oft ausgelöst? Haben uns nicht gerade die Regisseure des jungen deutschen Films zeigen wollen, wie wenig ideal solche Dorfgemeinschaften oft waren, welche Unmenschlichkeiten in ihnen geradezu gezüchtet wurden?

Es ist gewiß nicht wünschenswert, wenn alte Menschen Einsamkeit durch erhöhten Medienkonsum zu überwinden suchen. Nur, war denn wirklich das Leben der alten 
Menschen vor hundert oder zweihundert Jahren besser? Wahrscheinlich haben sie mehr gebetet, das kann sein. Ansonsten gibt es literarische Zeugnisse genug, die die aktive Generation auf ihre Versäumnisse und Hartherzigkeit der Elterngeneration gegenüber aufmerksam gemacht haben.

In der Diskussion um die Auswirkungen der Neuen Medien zeigt es sich immer wieder, daß sowohl die Gegner als auch die Befürworter mit Spekulationen arbeiten und dabei den betroffenen Menschen nicht sehr viel zutrauen. In den Bildschirmtext-Feldversuchen hat sich gezeigt, daß die Teilnehmer sehr gezielt ihre Informationen auswählen, im Durchschnitt fünf Minuten am Tag. Mir scheint, daß auch 54 Kanäle nicht den sonntäglichen Frühschoppen in Niederbayern verhindern werden. Weder Videotext noch Bildplatte, weder der Kommissar noch Dallas können die Attraktivität eines Münchner Biergarten erreichen. Und wer einmal an einem Sommerabend versucht hat, mit Brotzeitkorb und einer Schar Nachbarskinder dort einen Platz zu bekommen, der weiß, daß längst nicht die ganze Bevölkerung verstummt vor dem Fernsehgerät sitzt, womöglich jeder mit seinem eigenen niveaulosen Fluchtprogramm.

\subsection{Was sagt man dazu?}

Ich versuche, meine Umwelt und die Reaktionen meiner Mitmenschen gerade auf solche plausible Schlagworte wie Fluchtprogramm hin zu beobachten. So geschah es mir vor kurzem auf einer Dienstreise. Mir blieb keine Zeit mehr, eine Kleinigkeit zu essen und ich wollte mir mit einer Tafel Schokolade helfen. Am Bahnhofskiosk saß eine junge Verkäuferin, mit Lesen beschäftigt. Ich machte mich bemerkbar, indem ich sie grüßte und meinen Kaufwunsch vortrug. Keine Reaktion. Fasziniert bemerkte ich, daß sie mich überhaupt nicht wahrnahm, daß sie völlig versunken war in ihren Lesestoff, und das inmitten eines großstädtischen Bahnhofgetümmels. Die Abfahrt meines Zuges erlaubte kein weiteres Warten. Ich grüßte etwas lauter, worauf mein Gegenüber aus ihrer Lektüre auftauchte. Sie war tatsächlich völlig absorbiert in eines jener Romanheftchen, das wir zur Trivialliteratur zählen. Wie steht es dabei mit dem Bezug zur Wirklichkeit oder der Flucht daraus? Zeigt ein solches Beispiel nicht, daß es dieses Phänomen auch ohne Fernsehen gegeben hat und daß die Kulturtechnik "Lesen " nicht eo ipso besser ist? Es sind eben nicht alle Mängel in unserem Leben von den Medien verursacht noch diesen anzulasten.

Und was die sogenannte Nahraum-Kommunikation betrifft, so konnte ich ein paar Erfahrungen sammeln, was es heißt, in einer 25000 Einwohner zählenden HochhausVorstadt zu wohnen. Der Aufbau einer Stadtteilzeitung, die Arbeit einer kleinen Videogruppe hatten mehr direkte Kommunikations- und Begegnungserfolge als das städteplanerische Konzept. 25000 Menschen, die zum größten Teil wegen ihres Arbeitsplatzes zugezogen sind, bilden keine Dorfgemeinschaft. Die direkten Treffpunkte, die angebotenen Bildungs- und Freizeitveranstaltungen bedürfen oft eines eigenen Anstoßes. Eine Vorankündigung im Stadtteil-Fernsehen, eine Reportage über ein Gemeindefest, Informationen über Bebauungsplanungen, Kommentare zu aktuellen Ereignissen machen die Identifikation mit der oft anonymen und atomisierten Umwelt stufenweise leichter.

In diesem Zusammenhang verstehe ich auch die Aussage der Stellungnahme der Publizistischen Kommission der Deutschen Bischofskonferenz aus dem Jahr 1979, in der gerade solche Mitverantwortung für das Ganze betont wird: „Die Kirche begnügt sich nicht damit, Forderungen zu stellen oder Anregungen zur Bewältigung der kommenden Probleme zu geben. Vielmehr ist sie bereit, ihr Engagement in diese Bereiche ein- 
zubringen: Sie wird kommunikationspädagogische Maßnahmen und Initiativen unterstützen, die dazu dienen, den verantwortlichen Gebrauch der Medien einzuüben. Sie wird eigene Programme anbieten und an der Erarbeitung von Programmen mitwirken. Ihre pastoralen, sozialen und caritativen Dienste werden auch über die Neuen Medien den Bürgern zur Verfügung stehen. ${ }^{\boldsymbol{2 0 0}}$

\subsection{Orwell? - Nein!}

Während bei den vorangegangenen Überlegungen zu den Chancen und Risiken der Neuen Medien die Furcht vor allzu großem sozialen und menschlichen Schaden nicht übermäßig ist, möchte ich zum Schluß noch auf das wohl gravierendste Problem eingehen, daß sich besonders aus der Koppelung der neuen Kommunikationstechniken an datenverarbeitende Systeme ergibt.

Die Furcht vor dem Orwellschen 1984 dürfte wohl das größte Unbehagen und die stärkste Abwehr verursachen. Norbert Wieners Antwort auf solche Fragen ist, daß wir eine Gesellschaft haben müssen, die auf menschliche Werte gegründet ist. Er verweist damit den Schutz der Individualität des Bürgers als ethisches Problem an die Gesellschaft bzw. den Staat, daß er es löse. Das Konzept einer Gesellschaft, die auf menschliche Werte gegründet ist, muß sich darin ausdrücken, wie aufmerksam die flieBende Grenze zwischen Nützen und Schützen gezogen und auch beobachtet wird. Es müssen sowohl Gesetze geschaffen werden, die das Speichern von personenbezogenen Daten in Datenbanken begrenzen, als auch solche, die das Abfragen und Weitergeben solcher Daten auf das Notwendigste beschränkten. Totale Information macht zwar Verwaltungen effizienter, sie ist aber auch die Grundlage totaler Herrschaft. Die Qualität einer Gesellschaft wird sich darin zeigen, mit welchem Ernst sie diese Einschränkungen achtet. Gerade in Zeiten bedrohlicher Verunsicherung, „weil solche Angste - wie unbestimmt und unreflektiert auch immer - von Menschen erfahren werden, ist es eine pastorale Aufgabe der Kirche, den Menschen damit nicht allein zu lassen. Sie hat die Fragen, aus denen Angst spricht, ernsthaft und gründlich zu bedenken, die sich darin artikulierende Sorge nachzuempfinden und mitzutragen, nach Klärungen zu suchen und Wege zur Uberwindung aufzuzeigen ${ }^{\alpha 21}$.

Dies dürfte jedoch keine leichte Aufgabe sein. Die politischen und gesellschaftlichen Veränderungen scheinen kein Zurück mehr zuzulassen. „Lebende Systeme scheinen Gesetzmäßigkeiten zu unterliegen, die aber - da sie nicht ,einfach ' und ,streng ' sind - durch menschliches Handeln im Rahmen von Toleranzen modifiziert werden können. “22 Dabei ist die Befreiung zu einer besseren Kommunikation und Kooperation der Menschen untereinander das Kriterium christlichen Handelns.

Karl W. Deutsch stellt überraschend dar, wie die Resignation vor einer technischen Determiniertheit in schöpferisches Suchen nach neuen Lösungen aufgebrochen werden kann, wenn er sagt: „Die Organisation läuft ... gleichsam mit Scheuklappen, die sie sich selbst angelegt hat, und ein kumulativer Verlust an Sensibilität kann hinter einer Fassade von scheinbar reifer werdendem Verhalten in teilweiser Blindheit enden. Es scheint, daß dieser pathologische Aspekt im Lernprozeß der Erwachsenen den frühen Christen intuitiv geläufig gewesen ist. Ihre Aufforderung, die Menschen sollten,werden wie die Kinder', muß die Jünger der platonischen Philosophie, die den Zustand der Reife und Vollkommenheit priesen, schockiert haben. "23 $\mathrm{Er}$ meint wohl damit, sich nicht aus Angst und Resignation davon abhalten zu lassen, die Entwicklungen aufmerksam zu beobachten und sich auch nicht vorschnell auf bewährte Lösungen festzulegen. Das heißt, sich die Freiheit zu nehmen, schöpferisch nach dem noch Uner- 
probten zu suchen, um nicht in das Dilemma zu geraten, vor dem Karl Steinbruch ${ }^{24}$ warnt, nämlich aus falscher Philosophie in falsches Verhalten zu geraten oder sich aufgrund fehlender Philosophie der Ratlosigkeit zu überlassen. „Die Anfälle von tödlicher Ernsthaftigkeit, die uns als feierlich botschaftbringende und mit gewichtigen Idealen verbrämte Massenbewegungen bekannt sind ... haben gewöhnlich geistige Engstirnigkeit, Furcht, spartanische Strenge und sterile Konformität in ihrem Gefolge ... Die durch eine Krise ausgelösten Energien sind gewöhnlich auf rein zwedkbedingtes Handeln und auf die Nutzanwendung bereits bekannter Praktiken ausgerichtet. ${ }^{\alpha 25}$ Die Polarisierung in harte Fronten scheint den Prozeß kreativer und menschlicher Lösung nicht zu fördern.

\section{Anmerkungen:}

1 Vgl. Marshall McLuhan, Die magischen Kanäle, Düsseldorf-Wien 1968, S. 23.

2 Time The Weekly Newsmagazine, January 3, 1983, Vol. 121, No 1.

3 Günter Friedrichs/Adam Schaff (Hrsg.): Auf Gedeih und Verderb - Mikroelektronik und Gesellschaft, Bericht an den Club of Rome, Wien-München-Zürich 1982, S. 7.

4 Alexander King, Eine neue industrielle Revolution oder bloß eine neue Technologie? In: Friedrichs/Schaff, Auf Gedeih und Verderb, S. 23.

5 Wilhelm Steinmïller, Informationstechnologien und Informationssysteme: Folgen und Alternativen, in: Norbert Müllert (Hrsg.): Schöne elektronische Welt, Reinbek bei Hamburg 1982, S. 11.

- Claus Eurich, Der Verlust der Zwischenmenschlichkeit, in: Norbert Müllert (Hrsg.): Schöne elektronische Welt, S. $109 \mathrm{f}$.

7 Klaus Lenk, Informationstechnik und Gesellschaft, in: Friedrichs/Schaff, Auf Gedeih und Verderb, S. $300 \mathrm{f}$.

8 Warner Thorns, The Computer Society.

9 Alexander King, Mikroelektronik und globale Interdependenz, in: Friedrichs/Schaff, Auf Gedeih und Verderb, S. 352.

10 Zentralstelle Medien der Deutschen Bischofskonferenz (Hrsg.): Die Neuen Medien - Informationen, Fragen und Anregungen im Blick auf den Menschen und die Gesellschaft, Bonn 1982, S. 11. Materialien zur Medienpolitik Nr. 4.

11 Vgl. Die Neuen Medien, Informationen, Fragen, Anregungen, S. $9 \mathrm{f}$.

12 Eric Hoffer, Die Angst vor dem Neuen, Reinbek bei Hamburg 1968, S. 7. Originaltitel: The Ordeal of Change, 1952.

13 Karl W. Deutsd, Madht und Ohnmacht der Propheten - Vom schöpferischen Verhalten in der Geschichte, in: Die Welt Nr. 57, 8. 3. 1969, S. 1. Originaltitel: Nerves of Government. Úbersetzung: Politische Kybernetik. Modelle und Perspektiven, Freiburg 1969.

14 Socrates, Phaidrus.

15 Zitiert nach: Michael Schmolke, Die schlechte Presse, Münster 1971, S. 208.

16 Marshall McLuhan, Die magischen Kanäle, S. 293.

17 Claus Henninger, Auf dem Weg in die Grenzenlosigkeit. Die Raumfahrt verändert die Wirtschaft der Welt, in: Frankfurter Allgemeine Zeitung, 7. 5. 1983, Nr. 106, S. 13.

18 Claus Eurich, Der Verlust der Zwischenmenschlichkeit, S. 109.

19 Claus Eurich, Das verkabelte Leben. Wem schaden und wem nützen die Neuen Medien? Reinbek bei Hamburg 1982, S. 96.

${ }^{20}$ Stellungnahme zu medienpolitischen Fragen: Erklärung der Publizistischen Kommission der Deutschen Bischofskonferenz, Juni 1979, a. a. O., S. 35 f.

${ }^{21}$ Die Neuen Medien - Informationen, Fragen und Anregungen, a. a. O., S. 13.

22 Klaus Haefner, Der „Große Bruder", Chancen und Gefahren für eine informierte Gesellschaft, Düsseldorf-Wien 1980, S. 318.

${ }^{23} \mathrm{Karl}$ W. Deutsch, Macht und Ohnmacht der Propheten, a. a. O., S. I. 
${ }^{24}$ Karl Steinbuch, Vom Wesen der Information. Neue technische Möglidkeiten fordern eine grundsätzliche theoretische Klärung, in: Umschau 82, Heft 11, S. 363.

25 Eric Hoffer, Die Angst vor dem Neuen, a. a. O., S. 97 und S. 99.

\section{SUMMARY}

In our society, fear over the development of new media and the computerisation of mankind is growing. The anxiety over the isolation of man also increases as well as the one over the total control. Anxiety, uncertainty and pessimism are signs of changing times. Neither the specialists of the Club of Rome who study the changes of mankind today, nor politicans, philosophers or theologians can give solutions to reduce this fear for negative consequences of such new technologies. But still it is necessary through thinking and human action to recognise the dangers for mankind, and counteract accordingly.

\section{RESUME}

L'anxiété devant le développement de nouveaux Media et la Computerisation sont en croissance dans notre société: La peur de l'isolation de l'homme et du contrôle total augmente. Anxiéte, incertitude et pessimisme sont des attributs des temps changeants. $\mathrm{Ni}$ les experts qui se réunissent au Club of Rome pour étudier les périls auxquels l'humanité est exposée, ni les politiciens, philosophes ou théologiens peuvent offrir des solutions pour réduire la peur de conséquences négatives des nouvelles technologies. Malgré cela, il s’avère nécessaire de distinguer les dangers pour l'humanité par la réflection et l'action humaine et de réagir conformément.

\section{RESUMEN}

En nuestra sociedad crece el temor acerca del desarrollo de los nuevos media y la computarizacion de la humanidad. La ansiedad acerca del aislamiento del hombre crece tambien en relación a la posibilidad de un control total.

Ansiedad, pesimismo e inseguridad son signos de los tiempos que cambian. Ni los especialistas del Club de Roma, quienes estudian los cambios actuales de la humanidad, ni los políticos, filósofos o teólogos pueden dar soluciones para disminuir este temor que provocan las consecuencias negativas que comportan estas nuevas tecnologias. No obstante es necesario que a través del pensamiento y acciones del hombre se reconozcan los peligros para la humanidad y que se reaccione en consecuencia. 